\title{
Blob Transport in the Tokamak Scrape-off-Layer
}

\author{
D. A. D'Ippolito*1, J. R. Myra ${ }^{1}$, S. I. Krasheninnikov ${ }^{2}$, G. Q. Yu ${ }^{2}$, and A. Yu. Pigarov ${ }^{2}$ \\ ${ }^{1}$ Lodestar Research Corporation, 2400 Central Avenue P-5, Boulder, Colorado \\ ${ }^{2}$ University of California San Diego, La Jolla, California
}

\begin{abstract}
Key words tokamak, transport, SOL, convection, blobs
PACS 52.25.Fi, 52.35.Ra, 52.55.Dy, 52.55.Fa

Recent experimental evidence suggests the importance of fast radial plasma transport in the scrape-off-layer (SOL) of tokamaks. The outward transport appears to be convective rather than diffusive, extends into the far SOL, and can produce significant recycling from the main-chamber walls, partially bypassing the divertor. A plausible theoretical mechanism to explain this phenomenon is the radial transport of "blobs" of locally dense plasma created by turbulent processes. A related process is the inward transport of "holes" of reduced density plasma, which provides a mechanism for rapid inward transport of impurities. The blob model is also consistent with the spatial and temporal intermittency and the non-Gaussian statistics observed in the SOL plasma. This paper reviews the present status of blob theory, including analytic models and simulations, and discusses the preliminary comparisons of the blob model with experimental data.
\end{abstract}

\section{Introduction}

Recent experimental [1]-[12] and theoretical [13]-[21] work has led to new insights into the nature of radial transport in the scrape-off-layer (SOL) of tokamaks and other plasma confinement experiments. Measurements on both linear [5],[6] and toroidal [5]-[8] devices show that the radial transport in the SOL has a large convective component which is due to propagating, coherent structures. (The observation of such coherent structures is not new, dating back to the work reported in [22]). In tokamaks, the enhanced main chamber recycling caused by the interaction of the plasma flux with the walls can substantially reduce the efficiency of the divertor [4]. Modeling of the experimentally-inferred SOL transport on various tokamaks with the $2 \mathrm{D}$ edge transport code UEDGE shows that an outwards convective velocity $V_{c o n v}$ is needed to fit the data [15],[16],[21]. Moreover, an analysis of a series of DIII-D L-mode discharges having the same NBI input power but different averaged densities showed that $V_{\text {conv }}$ is an increasing function of density [see Fig. 1]. At high densities the cross-field transport becomes so fast that plasma flow into the divertor cannot compete with it [16],[21]. The modeling supports the hypothesis [23] that the tokamak density limit can be caused by cross-field plasma transport. This hypothesis has also been supported by recent 3D turbulence simulations [24]. It has also been shown that Edge Localized Modes (ELMs) on several tokamaks give rise to blob-like convective transport in the SOL [25]-[27], which diverts a significant amount of the particle and energy content away from the divertor. Thus, the nature of the convective SOL transport is important for understanding the boundary condition on core turbulence, the global particle and energy balance, mechanisms for impurity transport, and possibly the density limit.

The existence of propagating coherent structures is directly supported by Gas Puff Imaging [10]-[12] and Beam Emission Spectroscopy [7], [8] images showing localized regions with emission maxima and minima that move radially and poloidally. Probe data suggests that a substantial fraction of the SOL transport is caused by the propagation of these structures; the associated flux is intermittent in space and time and is described by non-Gaussian statistics [5]-[8]. (The skewness of the density and potential fluctuations is negative or zero in the core, positive in the SOL and tends to increase with distance from the separatrix.) The observed transport is outwards for concentrations of excess density ("blobs") and inwards for regions of reduced density ("holes") [8] in agreement with theory [21]. These structures have typical sizes of $1 \mathrm{~cm}$ for tokamaks [8] and $1-5 \mathrm{~cm}$ for various linear machines. Typical radial velocities are of order $10^{3} \mathrm{~m} / \mathrm{s}[5]-[8]$ and typical autocorrelation times

\footnotetext{
* Corresponding author: e-mail: dasd@lodestar.com, Phone: 303-449-9691 Fax: 303-449-3865
} 
are about $20 \mu s$ [7]. The universality and robustness of the phenomenon is shown by the fact that the statistics of the convective transport are similar on different machines [6].

The experimental work has been accompanied by the development of a theory of "blob transport" [13],[14], which ties together many of the observations. Blobs are observed to arise near the separatrix as a result of edge turbulence, probably as a result of the change in topology from closed to open field lines. Although turbulent in origin, they move as coherent (non-turbulent) objects. The term 'blob" refers to its shape seen in the 2D plane (see Fig. 2); in 3D it is a filament, localized perpendicular to the magnetic field but extended along B. A blob is created with concentrations of density, temperature, and vorticity higher than the surrounding plasma and convects these quantities from the near SOL outwards towards the colder and more tenuous far SOL.

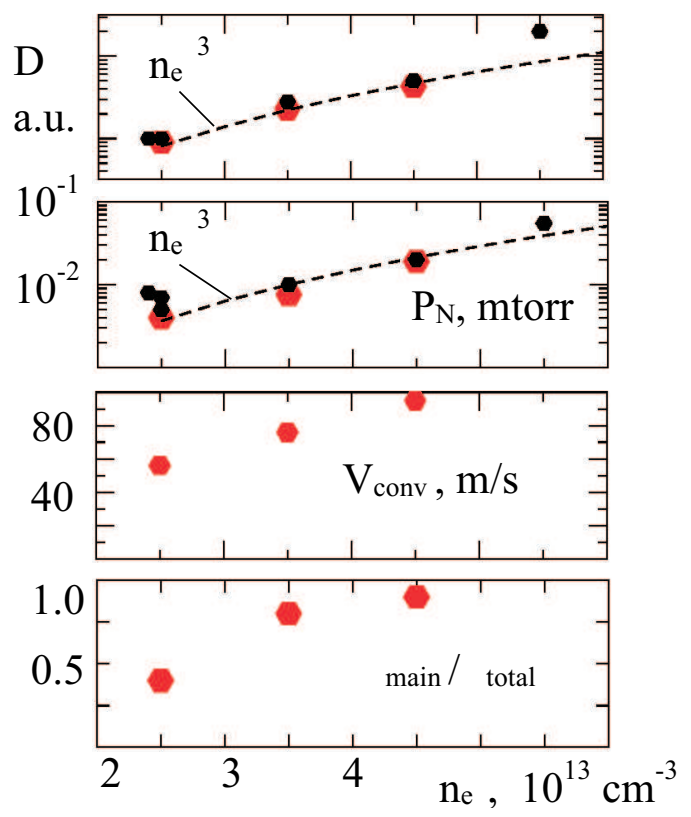

Fig. 1 Results of UEDGE modeling of DIII-D L-mode plasmas having different average densities. The large dots represent the modeling using the optimal outwards convective velocity $V_{\text {conv }}$; the small dots are the experimental data. [Reproduced from Refs. [16],[21]]

\section{separatrix}
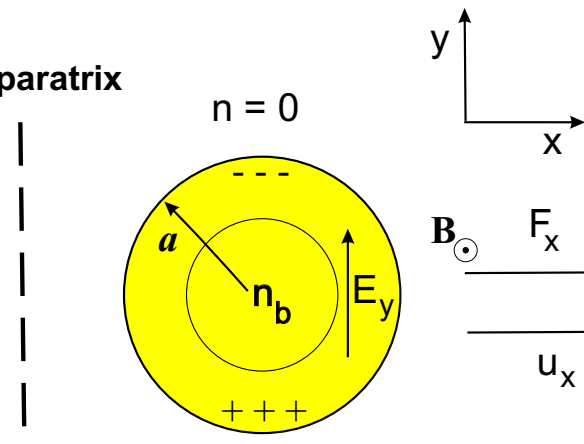

B. $F_{x}$

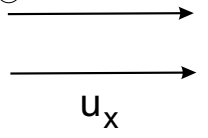

Fig. 2 Sketch illustrating the physics of blob charge polarization and transport in the SOL. The motion requires (1) a net species-summed outward force and (2) a sufficiently slow parallel current (polarization charge loss) into the sheaths.

Theory and simulations of blob transport agree qualitatively (and in some cases quantitatively) with the data. A simple analytic model of density blob transport has been studied [13],[14] and extended to include some results for blobs containing temperature and vorticity [14]. In the absence of a background plasma, the density blob model yields an analytic relation between the blob radial velocity $u_{x}$ and its spatial size, which represents an upper limit on the blob velocity observed in simulations. In this model, smaller blobs move faster and travel farther than larger ones, resulting in plasma profiles that resemble the experimental ones [14]. The radial transport predicted by the theory depends on the blob size distribution, so it is important to understand all processes that influence this distribution. As a first step, theory and computer simulations have studied "secondary instabilities" driven by curvature, velocity shear, and rotation [17]-[19]. These can result in blob fragmentation, thereby increasing the convective transport. The theoretical work provides estimates of typical blob sizes expected in the far SOL [18], [19]. The simulations also can treat the important effects of background plasma on the blobs. It is shown that the background plasma modifies the blob shape, velocity and stability in ways that are important for comparison with the experimental data. This work is still in progress, but shows encouraging qualitative agreement with the experiments.

The goal of this paper is to review recent theory and simulations of blob transport and to discuss the relation of this work to the experimental data. In Sec 2 we review the analytic theory of density blobs in the absence of a background plasma and describe the results of taking ensemble averages over the blob size distribution. In Sec. 3 
we describe the theory and simulations of blob secondary instabilities, introduce the concept of "essential stability," and discuss implications for the expected blob size. This section also discusses the effect of the background plasma on the blob shape, velocity, and stability. In Sec. 4 we give an assessment of the state of blob theory and a brief discussion of work in progress. A summary is given in Sec. 5.

\section{Analytic Blob Model}

In this section, we discuss an analytic model of density blobs in the absence of a background plasma. We assume that the vorticity has decayed and, for simplicity, that the temperature is uniform across the blob. The theory of blob convection stems from the simple observation that a density blob becomes charge polarized under the action of a net (species-summed) radial force [13], [14], as illustrated in Fig. 2. Here, the $x$ coordinate is in the direction of the major radius $R$, the $\mathrm{z}$ coordinate is taken along the direction of $\mathbf{B}$, and $\mathrm{y}$ is approximately in the poloidal direction at the outer midplane. Figure 2 depicts an isolated cylindrical blob (no background density) acted on by a radial force. The electrons and ions move in opposite directions due to the $\mathbf{F} \times \mathbf{B}$ drift, the blob charge distribution polarizes (if $\mathbf{b} \cdot \nabla \times \mathbf{F} \neq 0$ ), a poloidal electric field is created, and the resulting $\mathbf{E} \times \mathbf{B}$ drift moves the density blob outwards. (In the presence of a background density, numerical simulations show that the same mechanism moves a local "hole" of reduced density inwards [19], [21].) For the induced E field to survive on the transport time scale, charge flow along the field lines must be hindered by either sheath or plasma resistivity. At low density, we postulate that the blobs arise in the SOL due to the increase in resistivity upon crossing the separatrix into the open field line (sheath-dominated) region. Examples of forces which can produce blob convection include the curvature force [13],[14] in a toroidal device, the centrifugal force [13] for a rapidly rotating linear plasma device, and the "heutral wind force" [20].

The basic equations of the constant-temperature blob model are the vorticity and continuity equations for the potential $\varphi$ (or charge $\propto \nabla_{\perp}^{2} \varphi$ ) and the density $\mathrm{n}$. These equations can be written in dimensionless form as follows

$$
\begin{aligned}
& \frac{d}{d t}\left(n \nabla_{\perp}^{2} \varphi\right)+n \nu \nabla_{\perp}^{2} \varphi=\alpha n \varphi+2 \mathbf{b} \times \kappa \cdot \nabla n+\mathbf{b} \cdot \nabla \times(n \mathbf{a}), \\
& =\alpha n \varphi-\beta \nabla_{y} n-a_{x} \nabla_{y} n, \\
& \frac{d n}{d t} \equiv \frac{\partial n}{\partial t}+\mathbf{b} \times \nabla \varphi \cdot \nabla n=D \nabla_{\perp}^{2} n-\alpha n .
\end{aligned}
$$

Here, and throughout this paper, we use dimensionless units in which lengths are scaled to the gyroradius and frequencies to the cyclotron frequency, so that $L / \rho_{s} \rightarrow L, \Omega_{i} t \rightarrow t$, and $v / c_{s} \rightarrow v$. Equations (1) and (2) have been integrated along the field line, and the first term on the RHS of Eq. (1) was obtained by expanding the usual sheath term in the limit of small deviations from the Bohm sheath potential [see Ref. [14] for more details]. The LHS of Eq. (1) contains the inertial and viscous vorticity terms; the terms on the RHS represent the parallel flow of current into the sheaths, the perpendicular current due to the curvature drift and the effect of an arbitrary single-particle acceleration a. (The dimensionless acceleration is related to the dimensional force $\mathbf{F}$ by $\mathbf{F}=n m_{i} c_{s} \Omega_{i} \mathbf{a}$.) In the second form of Eq. (1), the derivatives were evaluated assuming that $\mathbf{a}=a_{x} \hat{\mathbf{e}}_{x}$ varies slowly compared to the density, viz. $\nabla_{y}\left(\ln a_{x}\right)<<\nabla_{y}(\ln n)$. Here, $\alpha=2 \rho_{s} / L_{\|}$and $\beta=2 \rho_{s} / R$ are the sheath and curvature parameters, respectively, $L_{\|}$is the parallel connection length and $R$ is the major radius of the tokamak. The terms on the RHS of the density equation, Eq. (2), describe the effects of particle diffusion and particle loss by parallel flow into the sheaths. For the analytic solution in this section, we set $\nu=0=D$ but these terms are retained in some of the numerical simulations described in Sec. 3.

An analytic solution of Eqs. (1) and (2) for the blob motion induced by the curvature force was given in [13],[14]. Consider a density profile of the general form

$$
n(x)=n_{b} \exp \left[-\frac{x^{2}}{2 x_{b}^{2}}-\frac{y^{2}}{2 y_{b}^{2}}\right]+n_{f}
$$

where $\left(x_{b}, y_{b}\right)$ denote the radial and poloidal dimensions of the blob, $n_{b}$ is the amplitude of the blob density perturbation, and $n_{f}$ is the background or "floor" density. The analytic solution is obtained by assuming small 
vorticity [neglect the LHS of Eq. (1)], $n_{f}=0$ and $\nu=0=D$. The parallel particle loss term can be included in the solution by letting $n_{b} \rightarrow n_{b} \exp (-\alpha t)$; we neglect this term in the remainder of this paper because it is small in the radial convection dominated limit. Balancing the sheath-conductivity term with the curvature term in Eq. (1) for the case $a_{x}=0$, one obtains a solution for the polarization potential, $\varphi=\Lambda \nabla_{y}(\ln n)$, and for the blob radial velocity $u_{x}=-\nabla_{y} \varphi$ :

$$
u_{x}=\frac{\Lambda}{y_{b}^{2}} \rightarrow \frac{\Lambda}{a^{2}}
$$

where $\Lambda=\beta / \alpha=L_{||} / R \propto q$ is the curvature force parameter and the second expression is obtained in the cylindrical blob limit $\left(a=x_{b}=y_{b}\right)$. Note that $y_{b}$ controls the radial $\mathbf{E} \times \mathbf{B}$ velocity because the charge polarization occurs in the poloidal direction.

The same relation holds for other forces satisfying $\mathbf{b} \cdot \nabla \times \mathbf{F} \neq 0$ with the appropriate redefinition of the force parameter $\Lambda$. For example, in the case of the neutral wind force [20], generated by the difference in friction felt by hot charge-exchange neutrals in the plasma edge and cold recycled wall neutrals, it can be shown that $\Lambda=\left(v_{n} / 2 c_{s}\right)\left(\nu_{i n} \tau_{\|}\right)$, where $v_{n}$ and $\nu_{i n}$ are the effective neutral velocity and ion-neutral collision frequency, and $\tau_{\|}=L_{\|} / c_{s}$. In the case of a general force $\left(a_{x} \neq 0\right.$ in Eq. (1)) we recover Eq. (4) with $\Lambda=L_{\|} a_{x}$.

Equation (4) implies that smaller blobs travel faster than larger ones and penetrate farther into the SOL (when the loss of particles by parallel flow is included in the model). Applied to an ensemble of blobs, this important scaling suggests that the density and particle flux profiles would be sensitive to the blob size distribution. To make this point more quantitative, a method of ensemble averaging was discussed in [14] and applied to the case of a power law distribution, $f(a)=1 / a^{p}$. It was found that the SOL density and flux profiles depend strongly on the value of $\mathrm{p}$ [see Fig. 2 of [14]]. We will return to this point in Sec. 4.

There are two processes that determine the distribution of blob sizes: (i) the "primary" edge/SOL instabilities and turbulent processes [28], [29] that create the blobs near the separatrix, and (ii) the "secondary" internal instabilities that cause blobs to fragment as they propagate [17]-[19]. The first topic is outside the scope of this paper, but we consider the second one in the next section.

\section{Secondary Instabilities and the Effect of Background Profiles}

The same forces that cause blob motion also give rise to instabilities on the global scale of the SOL ("primary" modes) and on the scale of the blobs ("secondary" modes). The nonlinear evolution of the turbulence arising from the primary instabilities is thought to create the blobs. The secondary instabilities (driven by the internal blob profiles) cause blob fragmentation. Since the smaller child blobs move faster than their parents, the net transport is increased by the instability when the growth rate is faster than the perpendicular convective transit time. Previous work has considered the sheath-interchange mode driven by curvature and the Kelvin-Helmholtz mode driven by radial velocity shear. Work is in progress to study the effect of rotational instabilities on the blob dynamics.

Simulations of blob instabilities have also investigated the effect of the background density profile on the blob shape, velocity, and stability. The results of these studies are important for comparing with the experimental data and are briefly summarized in the following sections.

\subsection{Secondary Instabilities and 'Essential Stability"}

To illustrate the competition between the blob instability and transport time scales, we first discuss sheathinterchange modes driven by curvature [17]-[19]. For poloidally-elongated blobs, the poloidal blob size is determined by the nonlinear evolution of modes with $k_{y} \neq 0$. Linearizing the basic equations of the blob model and making the "local approximation", we obtain the following dispersion relation [18] including the effects of dissipation:

$$
\gamma=-k_{y}^{2} D+\frac{k_{y}^{2}(\Lambda / L)}{1+k_{y}^{2} a_{s}^{2}}
$$


where $L=\left(\nabla_{x} \ln n\right)^{-1}$ is the scale length of the blob density profile at the point of maximum local density gradient, $D$ is the diffusion coefficient and $a_{s}=(\nu / \alpha)^{1 / 2}$ is the viscous "smoothing radius." For $D=0=$ $\nu$, Eq. (5) gives the local sheath-interchange growth rate $\left[\gamma=k_{y}^{2}(\Lambda / L)\right]$ first studied by Nedospasov [31]. Increasing the density floor $n_{f}$ has the effect of increasing $\mathrm{L}$ and reducing the growth rate. The dependence of blob stability on $n_{f}, D$, and $\nu$ was studied in some detail in [18],[19].

The instability growth time must be compared with the following characteristic equilibrium and transport times [see Fig. 3]: (i) the time to diffuse one blob radius, $\tau_{d}=a^{2} / D$, which is typically large; (ii) the time to convect one blob radius, $\tau_{c}=a / u_{x}=a^{3} / \Lambda$; and (iii) the time to convect to the wall, $\tau_{w}=\Delta x / u_{x}$. For simplicity, in discussing the transport estimates we consider cylindrical blobs with radius $a=x_{b}=y_{b}$ and replace $\tau_{w}$ by the time to convect a fixed number of blob radii. In [18] the separatrix-wall distance was assumed to be about 10 blob radii $(\Delta x=10 a)$. The corresponding transport rates are defined as $\gamma_{d}=1 / \tau_{d}, \gamma_{c}=1 / \tau_{c}, \gamma_{w}=1 / \tau_{w} \rightarrow \gamma_{c} / 10$ and note that they scale as $\gamma_{d} \propto 1 / a^{2}$ and $\gamma_{c} \propto 1 / a^{3}$.

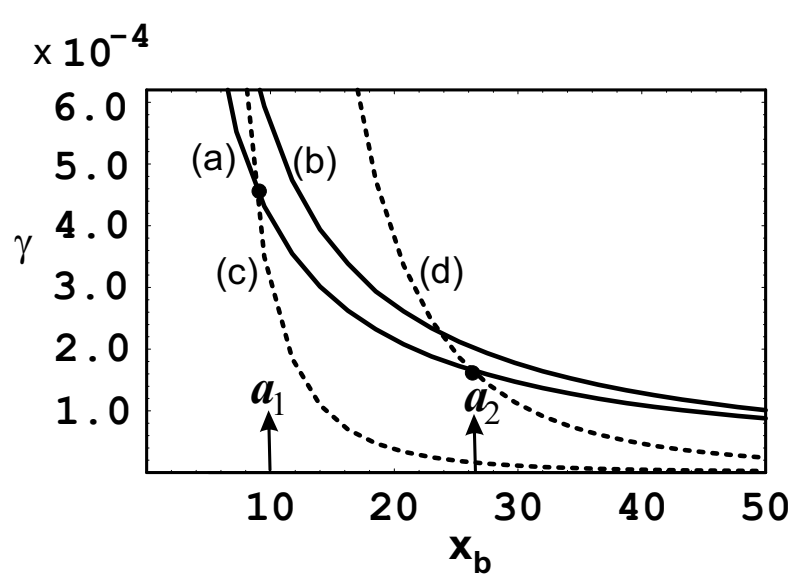

Fig. 3 Plots of the sheath-interchange growth rate and transport rates vs radial blob size $x_{b}$. Shown are (a) the maximum growth rate $\gamma_{\max }$ for the nonlocal numerical solution (lower solid curve), (b) $\gamma_{\max }$ for the local analytical solution (upper solid curve), (c) $\gamma_{c} / 10$ (lower dashed curve), and (d) $\gamma_{c}$ (upper dashed curve). The dots and arrows indicate the crossings of the stability and transport curves at $x_{b}=a_{1}$ and $a_{2}$, as explained in the text. [Reproduced from Ref. [18]]

Figure 3 illustrates the competition between the instability and transport rates as a function of radial blob size $x_{b}$. The growth rate $\gamma_{\max }\left(x_{b}\right)$ of the most unstable sheath-interchange mode is shown in the solid curves: (a) computed numerically including non-local effects, and (b) computed analytically from Eq. (5). The dashed curves show: (c) the blob transport rate to the wall, $\gamma_{c}\left(x_{b}\right) / 10$, and (d) the rate to transport one blob radius, $\gamma_{c}\left(x_{b}\right)$. This figure is explained in detail in [18]. Here, it suffices to recapitulate the significance of the crossing points, $a_{1}$ and $a_{2}$, which are defined by the conditions $\gamma=\gamma_{w}=\gamma_{c} / 10$ and $\gamma=\gamma_{c}$, respectively. Using Eq. (5) with $D=0=\nu$ and the expression previously given for $\gamma_{c}$, we obtain the approximate scalings $a_{1}=0.1\left(L / k_{y}^{2}\right)^{1 / 3}$ and $a_{2}=\left(L / k_{y}^{2}\right)^{1 / 3}$. The numerical results in Fig. 3 take into account the effects of dissipation and of using the most unstable $k_{y}$.

Cylindrical blobs with radius $a$ larger than $a_{2}$ move so slowly that they go unstable and break up before traveling one blob radius. These blobs will probably not be observed experimentally; we postulate that the radial autocorrelation time measured by probes can be estimated as $\tau=a_{2} / u_{x}\left(a_{2}\right)$, where the function $u_{x}(a)$ is defined in Eq. (4). This estimate agrees with the data to within a factor of 2. Blobs in the range $a_{1}<a<a_{2}$ will contribute to the radial transport while undergoing a cascade to smaller blob sizes. Blobs with $a<a_{1}$ will transport to the wall before going unstable. These blobs are "essentially stable" on the transport time scale [18]. It is reasonable to assume that the radial transport to the wall is due to the essentially stable blobs in the initial ensemble created near the separatrix. Taking $a_{1}$ as an upper bound on the size of the blobs that carry flux to the far SOL, we find a corresponding lower limit on the blob radial velocity, $u_{x}>u_{x}\left(a_{1}\right)=\Lambda / a_{1}^{2}$.

This discussion assumes that the linear instabilities will grow nonlinearly to large enough levels that the blobs actually break up. It also ignores the effect of sheared radial velocity. These limitations were removed by carrying out 2D simulations of blob dynamics, as discussed in the next section. 


\subsection{D Simulations of Curvature and Velocity-Shear Instabilities}

Several recent papers [17]-[19] have reported 2D simulations of blob dynamics that shed light on both the transport and stability of blobs. These simulations took the temperature to be constant and evolved only the vorticity and density equations. They show that the secondary instabilities grow nonlinearly to large enough amplitude to cause blob fragmentation.

The simplest model was used in [18]. Neglecting the inertial term in the vorticity equation (valid for large coherent structures), the simulation focused on the curvature-driven interchange instabilities that break up the larger blobs. This paper studied the stabilizing effects of diffusion, viscosity, and background density. It was shown that in the absence of large dissipation or background density, the instability grows to a high level and causes the blob to fragment. Even at moderate levels of viscosity $\left(a_{s} \approx a\right)$, the blobs were observed to bifurcate as they propagated (see Fig. 4). Consistent with the linear theory, it was found that the most unstable mode has $k_{y}>>k_{x}$. The sequence of frames in Fig. 4 shows the development of the instability for a case with finite viscosity but small background density. The bean shape in frame (b) is always seen in the simulations as the secondary instability develops and is probably associated with the background sheared flows (discussed below) seeding the instability. The end result in frame (d) is a bifurcation into two blobs with smaller $y_{b}$ than the original parent blob.
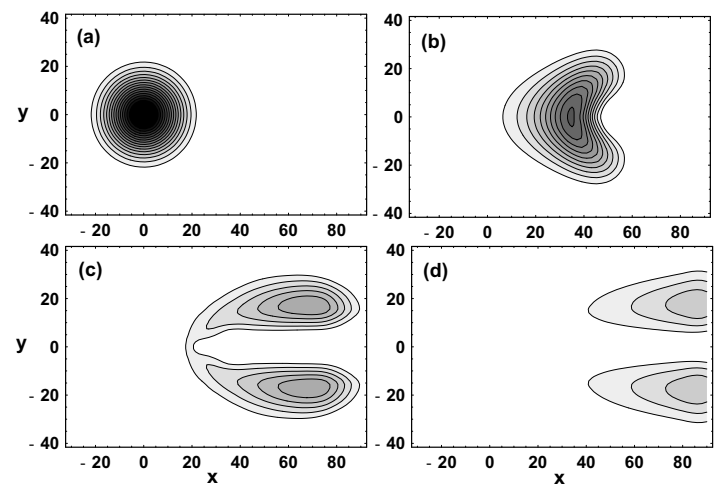

Fig. 4 Blob propagation showing weak instability and subsequent bifurcation for $n_{f}=0.01$. The four frames correspond to $t / \tau_{c}=0,6,9$ and 12 . [Reproduced from Ref. [18]]

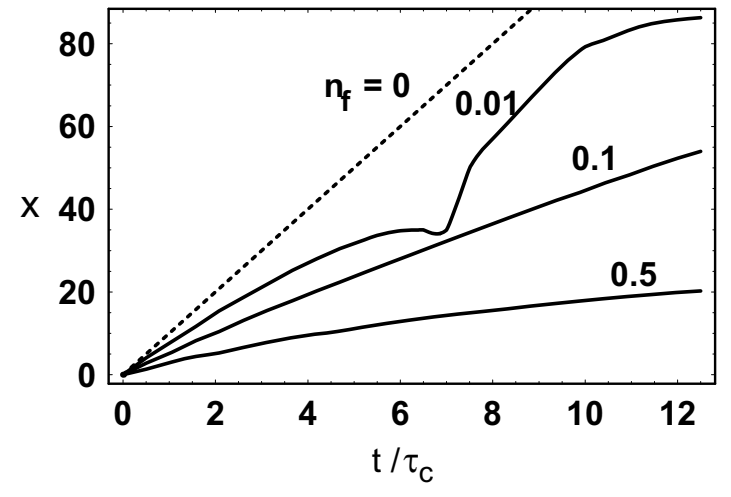

Fig. 5 Blob displacement $\boldsymbol{x}$ vs $t / \tau_{c}$ for the analytic result in Eq. (4) ( $n_{f}=0$, dashed line) and for cases having a fbor density of $n_{f}=0.01,0.1$ and 0.5 (solid curves). Note that the $n_{f}=0.01$ curve exhibits slow-down before the blob bifurcation at $t / \tau_{c} \approx 7$ and speed-up afterwards. [Reproduced from Ref. [18]]

Carrying out similar runs for various values of the background density [18] shows that, in general, the blob velocity depends on both its size $\left[y_{b}\right.$ in Eq. (4)] and on the amplitude of the blob relative to the background density. For the density profile in Eq. (3), the blob velocity depends on $n_{f} / n_{b}$ when this ratio is order unity, but the analytic result in Eq. (4) is recovered as $n_{f} / n_{b} \rightarrow 0$. The reason for the dependence of $u_{x}$ on the background density can be seen from the result that $\varphi=\Lambda \nabla_{y}(\ln n)$. Using Eq. (3) for the blob density and considering the region near $\mathrm{y}=0$, we find that $\varphi \propto\left[n_{b} /\left(n_{b}+n_{f}\right)\right]\left(y / y_{b}^{2}\right)$ and $u_{x} \propto\left[n_{b} /\left(n_{b}+n_{f}\right)\right] / y_{b}^{2}$.

This dependence is shown in Fig. 5. The solid lines trace the numerically-computed trajectories $x(t)$ of the same initial blob for three values of floor density $n_{f}$ with $n_{b}=1$. Also shown for comparison is a dashed line for the analytic $x(t)$ corresponding to Eq. (4). The average velocity computed from these trajectories drops as $n_{f} / n_{b}$ increases. The $n_{f}=0.01$ curve corresponds to the simulation shown in Fig. 4 , and we find that the blob exhibits slow-down before the bifurcation at $t / \tau_{c} \approx 7$ and speed-up afterwards. The slow-down at early times is associated with the deformation to larger $y_{b}$ observed in frame (b) of Fig. 4, and the speed up is associated with the reduced $y_{b}$ in frame (d). Thus, the polarization physics described by Eq. (4) is qualitatively correct and gives an upper bound on the blob velocity found in the simulations.

The 2D simulations show that several aspects of the blob dynamics are affected by the background density. In [18] it was shown that decreasing $n_{f} / n_{b}$ had the following effects: larger radial velocity [see Fig. 5], larger growth rate $\gamma_{S I}$ of the curvature-driven sheath-interchange instability, and greater deformation in blob shape. 
(The last two points are illustrated in Figs. 8 and 9 of Ref. [18].) It is not surprising that the radial velocity and instability growth rate scale the same way because $u_{x} \propto \nabla_{y} \ln n, \gamma_{S I} \propto \nabla_{x} l n n$, and the logarithmic derivative $\nabla \ln n$ decreases with $n_{f} / n_{b}$, as discussed after Fig. 5 .

The change in blob shape is more subtle, as illustrated in Figs. 6 and 7. The shape is modified by sheared flows $u_{x}(y)$ which arise when a background density is present. Figure 6 shows the density and potential $(\mathbf{E} \times \mathbf{B}$ flow $)$ contours for a blob propagating on a constant background density. The double-vortex structure of the potential contours leads to strongly-sheared flows near the maximum of the density gradient. These flows compress the density contours in the direction of the blob motion and spread out the contours behind the blob. Thus, the simulations [17]-[19] predict that a blob moving through a background density profile will have a steep leading edge and a trailing wake. We have simulated the time history $n(t)$ of the moving blob as would be seen by a probe at a fixed spatial point $(x, y)$. The result is shown in Fig. 7 for both the Gaussian blob in Eq. 3 using $n_{b}=0.5, n_{f}=0$ and for a numerical simulation [18] using $n_{b}=1, n_{f}=0.5$. The latter curve has the same characteristic shape as the time signals observed by probes [6]-[8],[27].

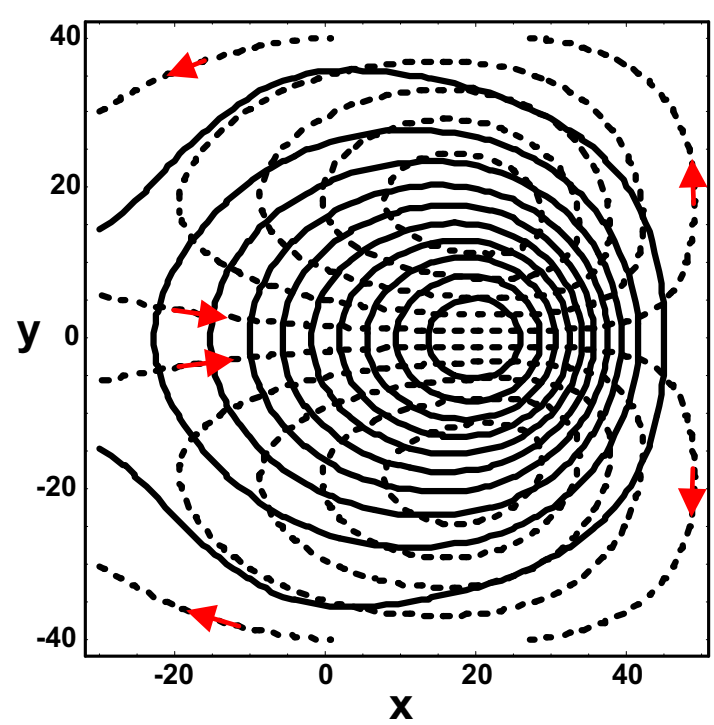

Fig. 6 Density (solid) and potential/fbw (dashed) contours in the lab frame for a blob propagating on a constant background density $\left(n_{f} / n_{b}=0.5\right)$. The interaction with the background density produces a sheared fbw pattern, which in turn gives a steep leading edge and trailing wake (also see Fig. 7)

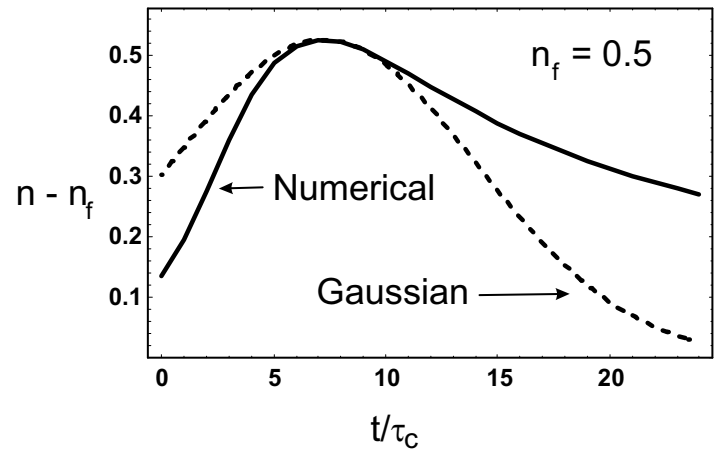

Fig. 7 Density blob time history for the numerical solution with a large fbor density $\left(n_{f}=0.5\right.$, solid line) and for a comparable Gaussian blob (dashed curve). The solid curve is qualitatively similar to experimental time traces obtained with probes.

The sheared velocity $u_{x}(y)$ can also drive the Kelvin-Helmholtz $(\mathrm{KH})$ instability [32] when the inertial term is retained in the vorticity equation [17],[19]. (The inertial term is important when considering small scale structures and/or rapid time scales.) For small blobs, the simulations show that the interaction of the dipolar (positive/negative) vortices leads to the formation of a thin spiral structure in the blob plasma density. The blob acquires a mushroom-like shape with a central core of propagating density while the periphery peels off and follows the flow contours. While the blob core will continue to propagate, it is likely that the peripheral material will be scattered. Thus, the KH instability can reduce the net blob transport and set a lower limit to the blob sizes contributing to the observed particle flux.

It was shown in [19] that the KH mode is the dominant instability for small blobs. This is due to the fact that the $\mathbf{E} \times \mathbf{B}$ convective nonlinearity in the vorticity inertial term is important at smaller space scales $\left(a \approx \rho_{s}\right)$, whereas the convective nonlinearity in the density equation is dominant at larger scales. Thus, the larger blobs $\left(a>>a_{c r i t}\right)$ are broken up by curvature-driven instabilities and the smaller ones $\left(a<<a_{c r i t}\right)$ by velocity-shear instabilities. The most stable blobs occur at intermediate scales ( $\left.a \approx a_{\text {crit }}\right)$; hence, $a_{\text {crit }}$ gives another way of estimating the most probable blob size. In [19] $a_{\text {crit }}$ is defined as the blob size at which the inertial, sheath, and 
curvature terms are all comparable in the vorticity equation. In the present notation, this condition is given by

$$
a_{c r i t}=\left(\Lambda L_{\|}\right)^{1 / 5}
$$

where $\Lambda$ is defined after Eq. (4).

Density holes are also subject to secondary instabilities. The KH instability of a density hole, with its evolving mushroom-like shape, is illustrated in Fig. 8. Notice that the hole propagates to the left, which is in the opposite direction to the imposed force and to the blob motion. It was suggested in [21] that the propagation of density holes from the far SOL towards the core can explain the rapid penetration of impurities seen in experiments. Neutral impurities that are ionized within the holes can be carried into the core plasma by the their inward convective motion.
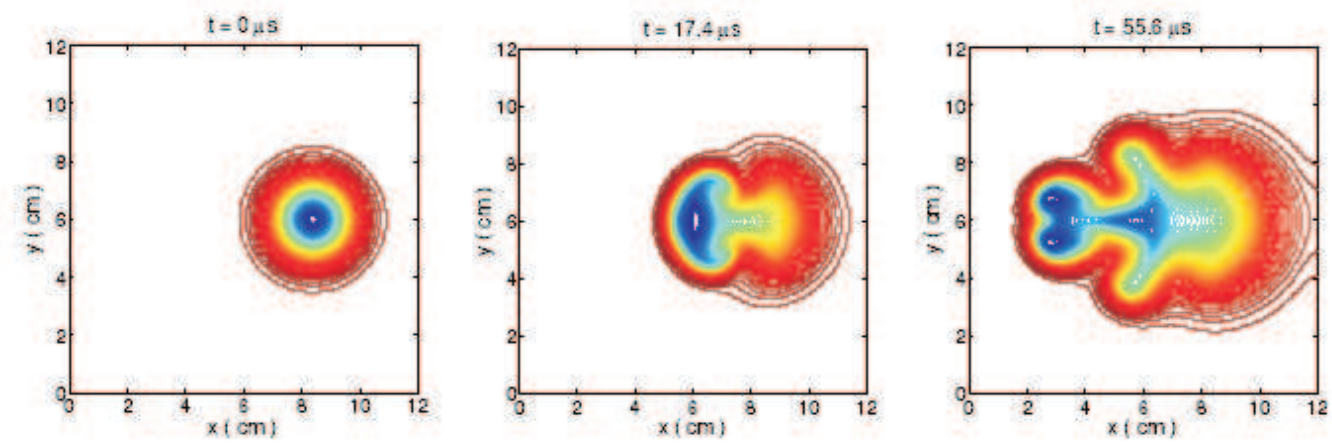

Fig. 8 Evolution of a density hole with $n_{b}=-n_{f} / 2$. Note that the hole moves in the opposite direction to the force and is unstable to the KH instability [Reproduced from Ref. [21]]

To summarize this section, 2D simulations with $T=$ const. show that the blob and hole dynamics are sensitive to instabilities driven by both curvature and velocity-shear $u_{x}(y)$. The growth rates depend on both the blob size and its amplitude relative to the background density. These secondary instabilities affect the blob size distribution and the net transport. When the model is generalized to include temperature variations, $T=T(x, y)$, a variety of additional physics is expected. In the next section, we discuss one example which may be relevant to the recent observations of ELM-induced blobs.

\subsection{Rotational Instabilities}

In previous sections, a constant electron temperature was assumed to simplify the computational models. In fact, the spatial variation of the temperature is an important factor in understanding the blob dynamics because of the sheath boundary condition in the SOL. Recall that quasineutrality in the SOL requires the formation of a Bohm sheath potential, $\Phi_{B} \approx 3 T_{e}$, to confine electrons, so that any spatial variation of the temperature leads to the development of electric fields and $\mathbf{E} \times \mathbf{B}$ drifts. For example, the internal blob temperature profile creates a centrally-peaked potential $\Phi(r)$ (assuming cylindrical blobs with radial coordinate $r$ ), a radial electric field, and blob rotation about its axis. If sufficiently fast, the rotation can modify both the radial velocity and the stability of the blob.

Strong blob rotation can spread out the polarization charge density $\rho \equiv-\nabla_{\perp}^{2} \Phi / 4 \pi$, thereby reducing the blob's internal electric field $E_{y}$ and radial velocity $u_{x}$ [33]. The presence of a background density ensures that $\rho \neq 0$ in the region of strong rotation. In 2D simulations, this effect can arise from the inertial term $(\alpha \mathbf{v} \cdot \nabla \rho)$ and from the viscous term $(\alpha \nu \rho)$. Work is in progress to evaluate the importance of this effect in modifying the blob transport [33].

In this section, we discuss the effect of rotation on the blob stability. We will assume that the background plasma temperature profile varies on a scale much longer than the blob radius, so that the background temperature gradient can be neglected when considering the internal blob rotation. (In general, the background temperature gradient would give rise to a net sheared poloidal motion of the blob.) We consider a "hot" blob that has not come into thermal equilibrium with the background plasma. It has an outwards pointing radial electric field $E_{r}$ that 
causes it to rotate about its axis. Again taking the blob to be uniform along $\mathbf{B}$, we can apply the rotational theory and variational principle developed many years ago for theta pinches [34] to study blob rotational instabilities [35]. The first interesting result of the theory is that both of the rotational terms (centrifugal $\propto \Omega^{2}$ and Coriolis $\propto$ $\Omega$, where $\Omega$ is the rotational frequency) are destabilizing when $E_{r}>0$ so that the instability drive is maximized; the only stabilizing effect is the usual FLR term. Second, one can estimate the scaling of the rotational instability growth rate $\gamma_{\boldsymbol{R}}$ and compare it with similar scalings for the curvature-driven sheath-interchange mode growth rate $\gamma_{S I}$ and the Kelvin-Helmholtz growth rate $\gamma_{K H}$. Neglecting FLR effects, taking $L_{n} \sim L_{T} \sim a$ and $k_{\perp} \sim k_{\theta} \sim m / a$ with $m$ the blob azimuthal mode number and $a$ the blob radius, and using the dimensionless notation of the present paper, we obtain the following scalings:

$$
\gamma_{R} \sim \frac{1}{a^{2}}, \quad \gamma_{S I} \sim \frac{m^{2}}{a^{3}}, \quad \gamma_{K H} \sim \frac{1}{a^{3}}
$$

Thus, the rotational growth rate is dominant for larger blobs $(a>>1)$ and lower mode numbers $m$. Comparing $\gamma_{R}$ and $\gamma_{S I}$ for a fixed blob size, we find that $\gamma_{R}>\gamma_{S I}$ for $m<m_{c r i t} \sim a^{1 / 2}$. It turns out that this is also the condition to neglect the curvature term in the vorticity equation. Finally, recall that the convection rate scales like $\gamma_{c} \sim 1 / a^{3}$, implying that convection is also slow compared to rotational instability growth for large blobs.

These scalings are summarized in Fig. 9. The shaded region satisfies the conditions that (i) $\gamma_{R}$ is the largest growth rate, and (ii) the rotational theory of [34] (neglecting curvature effects) is valid.

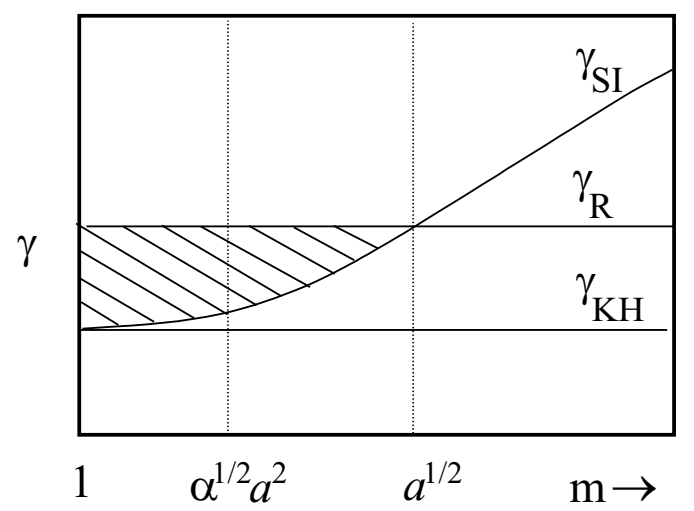

Fig. 9 Sketch of growth rate vs blob azimuthal model number $m$ for rotational $\left(\gamma_{R}\right)$, sheath-interchange $\left(\gamma_{S I}\right)$ and KelvinHelmholtz $\left(\gamma_{K H}\right)$ modes. The rotational instability is dominant in the shaded region.

In summary, our preliminary study [35] shows that large non-thermalized blobs are "essentially unstable" to rotational modes. Such modes may be a candidate to explain the fine-structure observed in the probe signals [25]-[27] produced by blobs associated with ELM crashes. Less fine structure is observed for smaller L-mode blobs, consistent with the basic scalings discussed here.

\section{Assessment of the Blob Model}

The blob model is appealing in its simplicity and intuitiveness. In the past few years, a great deal of theoretical work has been devoted to its study. It is appropriate to ask whether the model has been successful in explaining the experimental data. In this section, we describe areas of success, important unresolved issues, and areas for future work.

It has been shown that some qualitative features of the experiments emerge naturally from the blob transport model and simulations [13], [14], [17]-[21]. These include:

- a robust mechanism for convective transport and enhanced wall recycling in both toroidal [13], [14] and linear [20] machines

- two-scale density and particle flux profiles [14] 
- faster decay of SOL temperature than density [14]

- a critical particle flux for ionization-sustained equilibrium [14]

- a mechanism for inward transport of impurities [21]

- the shape of propagating pulses [17]-[19]

- intermittency and non-Gaussian statistics (see below)

- possible mechanisms for the density limit [21],[30] and for fine structure of ELMs [35]

The analytic blob model gives a mechanism of radial convective transport which is simple, robust, and gives order of magnitude estimates that agree with experiments. In a more quantitative approach, the density and flux profiles created by an ensemble of blobs are found to be sensitive to the blob size distribution because of the physics in Eq. (4). Taking a power law distribution, $f(a)=1 / a^{p}$ and carrying out an ensemble average over different size blobs, it is found that the SOL profiles are sensitive to the value of p [see Fig. 2 of Ref. [14]]. For large $p$ the small fast blobs dominate the transport and produce flat density and flux profiles which interact strongly with the wall. This behavior is qualitatively similar to the "Main Chamber Recycling Regime" on the Alcator C-Mod tokamak [4]. When ionization is included in the model, there is a synergy between blob transport and neutral recycling; the model [14] requires a minimum flux to the wall to obtain steady-state, similar to the observation in [4]. When the blob model is generalized to include temperature and vorticity, it can be shown that the vorticity and temperature decay faster than the density [14]. This feature also agrees with the experimental data [7], [8], [27].

The numerical simulations [17]-[19] generalize the blob model to include an interaction with the background density profile. As discussed in Sec. 3.2 this introduces a dependence of $u_{x}$ on the amplitude of the blob and also gives rise to a dipolar vortex sheared-flow pattern. One observable consequence of the sheared flow is the resulting shape of the propagating blob (steep leading edge, long trailing wake) [17]-[19] which agrees with the experimental data [6]-[8], [27]. The stability calculations yield a maximum blob size and an estimate of the autocorrelation time, which is in agreement with experiment [18].

The concept of blob propagation leads to transport which is intrinsically intermittent in time and space. However, one can make the argument more quantitative [36] by considering a regular train of density pulses and calculating the statistical moments. It has been shown that such a blob train has non-Gaussian statistics of the same sort as the experiment. In particular, the skewness is positive and of order unity. This analysis provides a possible point of contact between the blob model and the statistical database for the experiments. Work is underway to carry out this analysis for NSTX [36].

The blob model may provide a mechanism to explain the density limit. The role of convection in the density limit is suggested by C-Mod data [23]. Blob transport is dramatically enhanced at high density when electromagnetic effects are taken into account [30], and this effect can lead to a density limit in a reduced-2D electromagnetic model. The relationship of this work to the experimentally observed density limit in tokamaks is still unclear. In possibly related work, recent 3D turbulence simulations suggest that the strong increase of resistivity with density (at constant pressure) may also be an important factor in the density limit [24].

While there are many points of comparison between the blob model and experiments, a more quantitative analysis awaits further development of both the theory and the experimental diagnostics. The 2D simulation codes need to be generalized to include the spatial variation of the temperature, sheared flow (both poloidal and radial) and rotation. For $n_{e}$ approaching the density limit, it is certain that $3 \mathrm{D}$ simulations will be needed in order to compute the blob ballooning structure along the field lines. The blob creation problem must also be addressed using 3D turbulence codes such as BOUT [24],[28]. This study will require adequate resources of computer time and the development of new code diagnostics [37] to isolate the blob physics. Finally, the comparison of theory and experiments will require dedicated experiments on SOL transport in which a number of complementary diagnostics are used simultaneously to measure all of the parameters needed for the simulations.

\section{Summary and Conclusions}

Transport by 'blobs"(i.e. propagating coherent structures driven by charge polarization effects) provides a robust mechanism for explaining the observed intermittency and radial convection in turbulent SOL plasmas. Substantial 
theoretical progress has been made in recent years in understanding blob transport and stability, the interaction with the background density profile, and the origin of non-Gaussian statistics in the SOL [13]-[21],[30],[35],[36]. The theory has many points of contact with the experiment, but more realistic and comprehensive simulations are needed for quantitative comparison with the experimental data.

Blob motion is driven by a net radial force, which causes internal charge polarization and requires either sheath or plasma resistivity to inhibit the flow of charge along field lines. Examples of forces which have been observed to produce blobs in toroidal and linear experiments include the curvature [13],[14], centrifugal, and "neutral wind"forces [20]. The statistics of the resulting transport is remarkably universal across devices having different geometries, parameters, and basic force mechanisms [6]. The formation of such coherent structures and their transport is a subject of general importance in the field of plasma physics.

In this context, it is very interesting to note that similar enhanced density structures occur in ionospheric physics. It has been shown that "polar cap patches" are unstable to the gradient drift instability, producing smaller structures that propagate and break up due to secondary Kelvin-Helmholtz instabilities [38].

As discussed in Sec. 1, SOL convective transport has important implications for a fusion reactor. Blob transport allows the plasma and impurities to bypass the divertor; moreover, the importance of convective transport increases with density [see Fig. 1] and may also provide a mechanism for the density limit in tokamaks. The development of improved codes and numerical diagnostics will be necessary to make quantitative calculations of these effects.

Acknowledgements The authors wish to thank our experimental colleagues for many interesting discussions that motivated the theory discussed in this paper. This work was supported by U.S. Department of Energy (DOE) under grants DE-FG0397ER54392 and DE-FG03-00ER54568; however, such support does not constitute an endorsement by the DOE of the views expressed herein.

\section{References}

[1] M. Endler, H. Niedermeyer, L. Giannone et al., Nucl. Fusion 35, 1307 (1995).

[2] R. A. Moyer, R. D. Lehmer, T. E. Evans et al., Plasma Phys. Control. Fusion 38, 1273 (1996).

[3] R. Schneider et al., in Proceedings of the 17th IAEA Conference, Yokohama, Japan, 18-24 October, 1998, paper F1-CN69/THP2/05.

[4] M. Umansky, S. I. Krasheninnikov, B. LaBombard and J. L. Terry, Phys. Plasmas 5, 3373 (1998); B. LaBombard, M. V. Umansky, R. L. Boivin et al., Nucl. Fusion 40, 2041 (2000); B. LaBombard, R. L. Boivin, M. Greenwald et al., Phys. Plasmas 8, 2107 (2001).

[5] G. Y. Antar, S. I. Krasheninnikov, P. Devynck et al., Phys. Rev. Letters 87, 065001 (2001); G. Y. Antar, P. Devynck, X. Garbet, and S. C. Luckhardt, Phys. Plasmas 8, 1612 (2001).

[6] G. Y. Antar, G. Counsell, Y. Yu, B. LaBombard, and P. Devynck, Phys. Plasmas 10, 419 (2001).

[7] J. A. Boedo, D. Rudakov, R. Moyer et al., Phys. Plasmas 8, 4826 (2001); D. Rudakov, J. Boedo, R. Moyer et al., Plasma Phys. Control. Fusion 44, 717 (2002).

[8] J. A. Boedo, D. L. Rudakov, R. A. Moyer et al., Phys. Plasmas 10, 1670 (2003); J. A. Boedo, D. L. Rudakov, R. J. Colchin et al., J. Nucl. Mater. 313-316, 813 (2003).

[9] J. Neuhauser, D. Coster, H. U. Fahrbach et al., Plasma Phys. Control. Fusion 44, 855 (2002).

[10] S. Zweben, D. P. Stotler, J. L. Terry et al., Phys. Plasmas 9, 1981 (2002).

[11] J. L. Terry, S. J. Zweben, K. Hallatschek et al., Phys. Plasmas 10, 1739 (2003).

[12] S. J. Zweben, R. Maqueda, D. P. Stotler et al., to be published in Nucl. Fusion (2003).

[13] S. I. Krasheninnikov, Phys. Lett. A, 283, 368 (2001).

[14] D. A. D’Ippolito, J. R. Myra, and S. I. Krasheninnikov, Phys. Plasmas 9, 222 (2002).

[15] A. Yu. Pigarov, S. I. Krasheninnikov, T. D. Rognlien, M. J. Schaffer, and W. P. West, Phys. Plasmas 9, 1287 (2002).

[16] A. Yu. Pigarov, S. I. Krasheninnikov et al., J. Nucl. Mater. 313-316, 1076 (2003).

[17] N. Bian, S. Benkadda, J-V. Paulsen, and O. E. Garcia, Phys. Plasmas 10, 671 (2003).

[18] D. A. D'Ippolito and J. R. Myra, Lodestar Report LRC-03-92, May, 2003, to be published in Phys. Plasmas 10 (2003).

[19] G. Q. Yu and S. I. Krasheninnikov, to be published in Phys. Plasmas 10 (2003).

[20] S. I. Krasheninnikov and A. I. Smolyakov, Phys. Plasmas 10, 3020 (2003).

[21] S. I. Krasheninnikov, A. Yu. Pigarov, S. A. Galkin et al., in: Proceedings of the 19th IAEA Fusion Energy Conference, Lyon, France, 2002 (International Atomic Energy Agency, Vienna, 2003), paper IAEA-CN-94/TH/4-1.

[22] S. J. Zweben, Phys. Fluids 28, 974 (1985).

[23] M. J. Greenwald, Plasma Phys. Control. Fusion 44, R27 (2002).

[24] X. Q. Xu, W. M. Nevins, T. D. Rognlien et al., Phys. Plasmas 10, 1773 (2003). 
[25] M. Endler, S. Davies, I. Garcia-Cortes et al., in Proceedings of the 29th EPS Conference on Plasma Phys. and Control. Fusion, Montreux, Switzerland, 2002, edited by R. Behn and C. Varandas (European Physical Society, Paris, 2002), Europhysics Conference Abstracts Vol. 26B, paper O-3.24.

[26] B. Gonçalves, C. Hidalgo, M. A. Pedrosa, et al., Plasma Phys. Control. Fusion, 451627 (2003).

[27] J. A. Boedo, D. L. Rudakov, R. A. Moyer et al., submitted to Phys. Plasmas (2003).

[28] X. Q. Xu, W. M. Nevins, R. H. Cohen, J. R. Myra and P. B. Snyder, New J. Phys. 453.1 (2002).

[29] J. R. Myra, D. A. D’Ippolito, X. Q. Xu, and R. H. Cohen, Contrib. Plasma Phys. 40, 352 (2000); J. R. Myra, D. A. D'Ippolito, X. Q. Xu, and R. H. Cohen, Phys. Plasmas 7, 4622 (2000), and references therein.

[30] J. R. Myra, D. A. D'Ippolito, S. I. Krasheninnikov, and S. A. Galkin, in Proceedings of the 29th EPS Conference on Plasma Phys. and Control. Fusion, Montreux, Switzerland, 2002, edited by R. Behn and C. Varandas (European Physical Society, Paris, 2002), Europhysics Conference Abstracts Vol. 26B, paper O-3.23.

[31] A. V. Nedospasov, Fiz. Plazmy 15, 1139 (1989) [Sov. J. Plasma Phys. 15, 659 (1989)]; X. Garbet, L. Laurent, J.-P. Roubin, and A. Samain, Nucl. Fusion 31, 967 (1991).

[32] S. Chandrasekhar, Hydrodynamic and Hydromagnetic Stability (Oxford University Press, New York, 1961), Chap. XI; P. Pritchett, Phys. Fluids 30, 272 (1987).

[33] J. R. Myra, D. A. D'Ippolito, and S. I. Krasheninnikov, report in preparation (2003).

[34] J. P. Freidberg and L. D. Pearlstein, Phys. Fluids 21, 1207 (1978).

[35] D. A. D'Ippolito, J. R. Myra and D. A. Russell, 45th APS-DPP Meeting, Albuquerque, NM (2003), paper LP1.073.

[36] J. R. Myra, D. A. D’Ippolito, D. P. Stotler, S. J. Zweben, and R. Maqueda, 45th APS-DPP Meeting, Albuquerque, NM (2003), paper LP1.070.

[37] D. A. Russell, J. R. Myra, D. A. D’Ippolito, W. M. Nevins, X. Q. Xu, 45th APS-DPP Meeting, Albuquerque, NM (2003), paper LP1.071.

[38] P. N. Guzdar, N. A. Gondarenko, P. K. Chaturvedi and S. Basu, Radio Sci. 33, 1901 (1998); N. A. Gondarenko and P. N. Guzdar, Geophys. Res. Lett. 26, 3345 (1999) and Geophys. Res. Lett. 106, 24,611 (2001). 